\title{
Review
}

\section{Incidence, patient satisfaction, and perceptions of post-surgical pain: results from a US national survey}

Tong J. Gan

Ashraf S. Habib

Timothy E. Miller

William White

Duke University Medical Center, Durham, NC, USA

Jeffrey L. Apfelbaum

The University of Chicago, Chicago, IL, USA

\section{Address for correspondence:}

Tong J. Gan, MD, MHS, FRCA, Professor of

Anesthesiology, Vice Chair for Clinical Research,

Department of Anesthesiology, Duke University

Medical Center, DUMC 3094, Durham, NC 27710, USA.

Tel: +1 919681 4660; Fax: +1 919681 4698;

tong.gan@duke.edu

\section{Keywords:}

Post-surgical pain - Analgesia - Survey - Pain Patient satisfaction

Accepted: 21 October 2013; published online: 15 November 2013 Citation: Curr Med Res Opin 2013; 1-12

\begin{abstract}
Objective:

During the past two decades, professional associations, accrediting bodies, and payors have made postsurgical pain treatment a high priority. In light of the disappointing findings in previous surveys, a survey was conducted to assess patient perceptions and characterize patient experiences/levels of satisfaction with post-surgical pain management.
\end{abstract}

\section{Research design and methods:}

Survey included a random sample of US adults who had undergone surgery within 5 years from the survey date. Participants were asked about their concerns before surgery, severity of perioperative pain, pain treatments, perceptions about post-surgical pain and pain medications, and satisfaction with treatments they received.

Results:

Of the 300 participants, 86\% experienced pain after surgery; of these, $75 \%$ had moderate/extreme pain during the immediate post-surgical period, with $74 \%$ still experiencing these levels of pain after discharge. Post-surgical pain was the most prominent pre-surgical patient concern, and nearly half reported they had high/very high anxiety levels about pain before surgery. Approximately $88 \%$ received analgesic medications to manage pain; of these, $80 \%$ experienced adverse effects and 39\% reported moderate/severe pain even after receiving their first dose.

\section{Study limitations:}

Key study limitations include the relatively small population size, potential for recall bias associated with the 14-month average time delay from surgery date to survey date, and the inability to account for influences of type of surgery and intraoperative anesthetic/analgesic use on survey results.

\section{Conclusions:}

Despite heightened awareness and clinical advancements in pain management, there has been little improvement in post-surgical analgesia as measured by this survey of post-surgical patients.

\section{Introduction}

According to statistics from the Centers for Disease Control and Prevention, $\sim 100$ million surgical procedures are performed in the US each year ${ }^{1}$. Of these, $\sim 60 \%$ are conducted in an ambulatory setting ${ }^{1,2}$, with up to $80 \%$ of patients experiencing pain after their procedure ${ }^{3,4}$.

Adequate treatment of acute pain improves clinical and economic outcomes ${ }^{3}$ and, during the past 2 decades, there has been increased focus on the need for 
better post-surgical pain management. Several guidelines published by government-sponsored healthcare agencies and non-governmental clinical societies outline strategies for improving pain management $t^{5-8}$. In recognition of the pervasive inadequacy of pain management in the surgical setting, guidelines commissioned by the Office of the Forum for Quality and Effectiveness in Health Care of the Agency for Healthcare Research and Quality (AHRQ; formerly the Agency for Health Care Policy and Research [AHCPR]) were first published in $1992^{5}$. In 2002, the Veterans Health Administration formally recognized pain as the fifth vital sign and published guidelines for post-operative pain management ${ }^{7}$. In 2001, the Joint Commission pain management standards went into effect for all accredited healthcare institutions in the US ${ }^{8}$. Practice guidelines for pain management in the perioperative setting, first published by the American Society of Anesthesiologists in $1995^{9}$, were updated in $2004^{10}$, and again in $2012^{6}$.

Pain management guidelines appear to have had little impact on practice patterns or improvement in pain control for patients. One year after the AHCPR guidelines were released, Warfield and $\mathrm{Kahn}^{4}$ conducted a national survey to assess the status of acute pain management in US hospitals $(n=300)$ and another to assess the attitudes of adults $(n=500)$ about post-surgical pain management. The hospital survey consisted of 36 questions concerning the status of current and future acute pain management programs; the survey of adults consisted of 36 questions regarding respondents' attitudes and experiences with post-operative pain and its management. Of the 300 hospitals surveyed, only $46 \%$ had written guidelines or a formal pain management program in place. Of the 500 participants surveyed, 27\% (135) had undergone surgery during the past 5 years; of these, $77 \%$ reported pain after surgery, with $61 \%$ experiencing moderate-to-extreme pain. One shortcoming of this survey was that patients were not stratified as inpatients or outpatients. About 10 years later, Apfelbaum et al. ${ }^{3}$ conducted a similarly designed survey to assess whether pain management practices had improved during the decade after the Warfield survey. When Apfelbaum et al. surveyed 250 adults who had undergone surgery within the previous 5 years, their results showed that the prevalence and characteristics of post-surgical pain had not improved over the course of the previous 10 years. In their survey, 82\% (206/250) reported pain after surgery; of these patients, $86 \%$ experienced moderate-to-severe pain, with similar results observed for inpatient and outpatient sub-groups.

In light of the increased focus on acute pain management and disappointing findings from these previous surveys, we sought to conduct a contemporary assessment of patient perceptions of post-surgical pain and to characterize their experiences and levels of satisfaction with post-surgical pain management.

\section{Patients and methods}

The study was approved by the Duke University Medical Center Institutional Review Board; patient consent requirements were waived. In the recruiting effort for this institutional study, 10,235 US physicians were randomly selected and asked to provide patients with a toll-free phone number to call to participate in the research. This process was performed using a random selection software program. The physician sample was designed to be representative of the US physician population in terms of geographic regions and medical specialties. Each patient who completed the survey received a $\$ 20$ incentive and the referring physician received a $\$ 10$ incentive. Physicians were allowed to refer a maximum of three patients. Adults of 18 years of age or older who underwent surgery within a period of no longer than 5 years from the date of the survey were eligible to participate, similar to the design of the previous study reported by Apfelbaum et al. ${ }^{3}$. Enrollment was closed when the number of patients reached 300.

Survey participants were asked a pre-defined set of questions about their pre-operative and post-operative pain experiences (Appendix). Questions from previously conducted surveys were included ${ }^{3,4}$, along with modified and new questions written by the investigators of the current study. The new questions were tested in a sample of patients to establish face and content validity. Participants were asked about their concerns before surgery, about pre- and post-operative pain levels, perioperative medications received, pain levels following analgesic administration, non-pharmacologic pain management techniques, satisfaction with pain management, analgesic-related adverse events, perioperative pain consultations, pain threshold levels, and pain-related anxiety.

The data were stratified according to surgical setting (inpatient vs outpatient) and summarized using descriptive statistics, with percentages calculated based on the total number of patients who answered each question. Those who had surgical procedures at a hospital as an outpatient, or at a doctor's office, outpatient clinic, or freestanding surgical center were classified as 'outpatients'. KruskalWallis or Wilcoxon 2-sample tests were performed, as appropriate, to assess the degree of association between parameters of interest.

\section{Results}

A total of 300 patients were included in this study. Demographic data are presented in Table 1. Approximately half of the respondents underwent surgery in an outpatient setting. The mean time from end of surgery to survey date was $\sim 14$ months. 
Table 1. Demographics of survey respondents.

\begin{tabular}{cccc}
\hline Parameter & $\begin{array}{c}\text { Inpatient } \\
(n=146), n(\%)\end{array}$ & $\begin{array}{c}\text { Outpatient }^{\mathrm{a}} \\
(n=154), n(\%)\end{array}$ & $\begin{array}{c}\text { Total } \\
(n=300), n(\%)\end{array}$ \\
\hline Age, years & & & \\
$18-39$ & $35(24)$ & $64(42)$ & $99(33)$ \\
$40-54$ & $45(31)$ & $46(30)$ & $91(30)$ \\
$55-64$ & $26(18)$ & $29(19)$ & $55(18)$ \\
$\geq 65$ & $40(27)$ & $15(10)$ & $55(18)$ \\
Sex & & & \\
Male & $49(34)$ & $56(36)$ & $105(35)$ \\
Female & $97(66)$ & $98(64)$ & $195(65)$ \\
Race & $115(79)$ & $115(75)$ & $230(77)$ \\
White & $11(8)$ & $15(10)$ & $26(9)$ \\
Hispanic & $4(3)$ & $6(4)$ & $10(3)$ \\
Black & $9(6)$ & $10(6)$ & $19(6)$ \\
Asian & $6(4)$ & $8(5)$ & $14(5)$ \\
Other &
\end{tabular}

alncludes patients with surgical procedures performed in a hospital as outpatients, doctor's office, an outpatient clinic, or a freestanding surgery center.

Table 2. Patient responses regarding concerns prior to surgery ${ }^{\mathrm{a}}$.

\begin{tabular}{|c|c|c|c|}
\hline Concern & $\begin{array}{c}\text { Inpatient } \\
\left(\begin{array}{c}n=146) \\
n(\%)\end{array}\right.\end{array}$ & $\begin{array}{c}\text { Outpatient } \\
(n=154), \\
n(\%)\end{array}$ & $\begin{array}{c}\text { Total } \\
(n=300) \\
n(\%)\end{array}$ \\
\hline Pain after surgery & $122(84)$ & $118(77)$ & $240(80)$ \\
\hline $\begin{array}{l}\text { Whether surgery would } \\
\text { improve condition }\end{array}$ & $93(64)$ & $94(61)$ & $187(62)$ \\
\hline Full recovery from surgery & $84(58)$ & $67(44)$ & $151(50)$ \\
\hline Pain during surgery & $73(50)$ & $62(40)$ & $135(45)$ \\
\hline $\begin{array}{l}\text { Treatment by healthcare } \\
\text { professionals }\end{array}$ & $65(45)$ & $39(25)$ & $104(35)$ \\
\hline No concerns & $10(7)$ & $17(11)$ & $27(9)$ \\
\hline
\end{tabular}

${ }^{\mathrm{a}}$ Patients could choose more than one concern.

Before surgery, post-surgical pain was the most prominent concern among patients surveyed, with $80 \%$ of respondents expressing concern about this issue (Table 2). When queried regarding anxiety about surgery-related pain before their surgery (response choices were 'very high', 'high', 'neutral', 'low', or 'very low'), $53 \%$ (78/146) of respondents who had inpatient surgery reported 'high' or 'very high' levels of anxiety compared with $40 \%(61 / 154)$ of those who had undergone surgery in an outpatient setting. Most patients (72\% of inpatients; $70 \%$ of outpatients) classified their pain threshold as 'high' or 'very high'.

Overall, about half of the respondents reported having pain before surgery, and $86 \%$ experienced pain after surgery (Table 3 ). Of the patients with pain prior to surgery, $38 \%(60 / 159)$ were taking analgesic medications preoperatively. The intensity of the post-surgical pain was described as moderate, severe, or extreme by $75 \%$ of the patients with some post-surgical pain ( $65 \%$ of all patients); a slightly larger proportion (79\%) of the inpatient subgroup than the outpatient sub-group (72\%) who had pain described its intensity as moderate-to-extreme. The survey item assessing post-discharge pain was answered by 225 respondents, of whom $84 \%$ reported some pain after hospital discharge. The intensity of pain was reported as moderate, severe, or extreme by $74 \%$ of those reporting pain (62\% of all respondents), with inpatient and outpatient sub-group rates of $71 \%$ and $78 \%$, respectively.

Approximately 88\% (225/257) of all respondents with post-surgical pain received analgesic medications after surgery, $93 \%(124 / 134)$ of the inpatient group vs $82 \%$ (101/ 123 ) of outpatients. Of the inpatients who reported receiving medication for post-surgical pain, 57\% (71/124) received their medication on a timed schedule, 23\% (28/ 124) received it via a patient-controlled device, $16 \%$ (20/ 124) had to ask for pain medication when needed, and $4 \%$ (5/124) had analgesia provided via an epidural device or nerve block; $95 \%$ of the outpatient sub-group received their pain medication on a timed schedule. Opioids and, in particular, hydrocodone, oxycodone, and morphine, were by far the most frequently administered analgesics for post-surgical pain in both the inpatient and outpatient settings (Table 4). Hydrocodone was used in a higher proportion of outpatient responders (46/101 [46\%] vs 40/124 [32\%] of inpatient responders); morphine was used in a higher proportion of inpatients (16\% of inpatient vs 3\% of outpatient responders). About 39\% (73/186) of patients reported moderate-to-severe pain, even after receiving the first dose of analgesic medication, a rate that was similar across the inpatient and outpatient sub-groups. Overall, $79 \%$ of those who received pain medications experienced at least one adverse effect. The most frequently reported adverse effects in both inpatients and outpatients were those typically observed with opioid analgesics: drowsiness, constipation, and nausea (Table 5).

Of the 225 patients who responded, 135 (60\%) reported that non-pharmacologic pain management strategies were used during post-surgical recovery. Application of cold, application of heat, and prescribed exercise regimens were the most common non-pharmacologic methods. Exercise regimens were prescribed twice as frequently for inpatients $(31 \%$ of inpatient vs $15 \%$ of outpatient respondents).

The vast majority of patients in the survey (90\%) reported being somewhat or very satisfied with their perioperative pain management. About two thirds of patients in the inpatient and outpatient sub-groups said they were very satisfied with their pain management and only $2 \%$ of each sub-group said they were very dissatisfied. About 75\% of respondents received counseling about pain from a healthcare professional before and again after surgery. Overall, surgeons and nurses were the most likely healthcare professionals to provide perioperative counseling about pain, with nurses more likely to give pre-operative pain counseling in the inpatient setting and surgeons more likely in the outpatient setting. After surgery, nurses were 
Table 3. Presence of pain and maximum intensity of pain experienced.

\begin{tabular}{lrrr}
\hline Variable, $n(\%)$ & Inpatient $(n=146)$ & Outpatient $(n=154)$ & Total $(n=300)$ \\
\hline Patients reporting pre-operative pain & $85 / 146(58.2)$ & $74 / 154(48.1)$ & $159 / 300(53.0)$ \\
Slight & $7(8.2)$ & $15(20.3)$ & $22(13.8)$ \\
Moderate & $20(23.5)$ & $25(33.8)$ & $45(28.3)$ \\
Severe & $58(68.2)$ & $34(46.0)$ & $92(57.9)$ \\
Patients reporting post-operative pain & $134 / 146(91.8)$ & $123 / 154(79.9)$ & $257 / 300(85.7)$ \\
Slight & $28(20.9)$ & $35(28.5)$ & $63(24.5)$ \\
Moderate & $63(47.0)$ & $52(42.3)$ & $115(44.8)$ \\
Severe & $27(20.2)$ & $31(25.2)$ & $58(22.6)$ \\
Extreme & $16(11.9)$ & $5(4.1)$ & $21(8.2)$ \\
Patients reporting pain after first analgesic medication administered & $102 / 124(82.3)$ & $84 / 101(83.2)$ & $186 / 225(82.7)$ \\
Slight & $61(59.8)$ & $52(61.9)$ & $113(60.8)$ \\
Moderate & $31(30.4)$ & $27(32.1)$ & $58(31.2)$ \\
Severe & $10(9.8)$ & $5(6.0)$ & $15(8.1)$ \\
Patients reporting pain after discharge & $109 / 124(87.9)$ & $80 / 101(79.2)$ & $189 / 225(84.0)$ \\
Slight & $32(29.4)$ & $18(22.5)$ & $50(26.5)$ \\
Moderate & $48(44.0)$ & $39(48.8)$ & $87(46.0)$ \\
Severe & $20(18.4)$ & $20(25.0)$ & $40(21.2)$ \\
Extreme & $9(8.3)$ & $3(3.8)$ & $12(6.4)$ \\
\hline
\end{tabular}

Table 4. Post-surgical analgesics used.

\begin{tabular}{lccc}
\hline Analgesic, $n$ (\%) & $\begin{array}{c}\text { Inpatient } \\
(n=124)\end{array}$ & $\begin{array}{c}\text { Outpatient } \\
(n=101)\end{array}$ & $\begin{array}{c}\text { Total } \\
(n=225)^{\mathrm{a}}\end{array}$ \\
\hline Morphine & $20(16)$ & $3(3)$ & $23(10)$ \\
Meperidine & $4(3)$ & $1(1)$ & $5(2)$ \\
Hydromorphone & $8(6)$ & $2(2)$ & $10(4)$ \\
Hydrocodone & $18(15)$ & $14(14)$ & $32(14)$ \\
Hydrocodone + acetaminophen & $22(18)$ & $32(32)$ & $54(24)$ \\
Oxycodone & $16(13)$ & $7(7)$ & $23(10)$ \\
Oxycodone + acetaminophen & $21(17)$ & $24(24)$ & $45(20)$ \\
Propoxyphene & $1(0.8)$ & 0 & $1(0.4)$ \\
Propoxyphene + acetaminophen & $3(2)$ & 0 & $3(1)$ \\
Codeine + acetaminophen & $9(7)$ & $6(6)$ & $15(7)$ \\
Ketorolac & $5(4)$ & $3(3)$ & $8(4)$ \\
Aspirin & $1(0.8)$ & 0 & $1(0.4)$ \\
lbuprofen & $6(5)$ & $2(2)$ & $8(4)$ \\
Acetaminophen & $8(6)$ & $3(3)$ & $11(5)$ \\
\hline
\end{tabular}

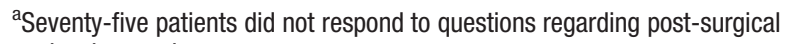
analgesics used.

the most likely healthcare professionals to provide pain counseling in both the inpatient and outpatient settings. Surprisingly, only $24 \%$ of patients reported receiving preoperative pain counseling from an anesthesiologist, and only $3 \%$ received post-operative counseling from an anesthesiologist.

Responses to questions regarding patients' perceptions about post-surgical pain and pain medications showed that $84 \%(251 / 300)$ felt post-surgical pain was necessary, and $94 \%(288 / 300)$ thought it was okay to complain about their pain. Only $12 \%(35 / 300)$ admitted that they had ever cancelled or delayed a surgical procedure for fear of pain. When asked about the route of pain medication administration that provided the best pain relief, 31\% $(92 / 300)$ selected the intravenous route, $17 \%(52 / 300)$ chose oral tablet administration, and 10\% (29/300)
Table 5. Adverse effects.

\begin{tabular}{lrrr}
\hline Adverse effect & $\begin{array}{r}\text { Inpatient } \\
\left(\begin{array}{l}n=124), \\
n(\%)\end{array}\right.\end{array}$ & $\begin{array}{r}\text { Outpatient } \\
\left(\begin{array}{l}n=101), \\
n(\%)\end{array}\right.\end{array}$ & $\begin{array}{c}\text { Total } \\
\left(\begin{array}{l}n=225), \\
n(\%)\end{array}\right.\end{array}$ \\
\hline Drowsiness & $70(56)$ & $56(55)$ & $126(56)$ \\
Constipation & $47(38)$ & $31(31)$ & $78(35)$ \\
Nausea & $31(25)$ & $32(32)$ & $63(28)$ \\
Dizziness & $19(15)$ & $20(20)$ & $39(17)$ \\
Itching & $18(15)$ & $16(16)$ & $34(15)$ \\
Sleeplessness & $18(15)$ & $10(10)$ & $28(12)$ \\
Mood changes & $17(14)$ & $10(10)$ & $27(12)$ \\
Confusion & $17(14)$ & $9(9)$ & $26(12)$ \\
Vomiting & $9(7)$ & $15(15)$ & $24(11)$ \\
Difficulty urinating & $6(5)$ & $1(1)$ & $7(3)$ \\
Slow or troubled breathing & $4(3)$ & $3(3)$ & $7(3)$ \\
\hline
\end{tabular}

${ }^{\text {aS }}$ eventy-five patients did not respond to questions regarding analgesicrelated adverse events.

chose the elastomeric pain pump. About 92\% (275/300) believed pain medications cause adverse effects, and, given a choice between narcotic and non-narcotic pain medications, more patients stated a preference for non-narcotic pain medications $(57 \%, 172 / 300)$ than narcotic medications $(36 \%, 109 / 300)$. Conversely, patient concerns regarding addiction were not highly prevalent-30\% (89/300) of patients had concerns about becoming addicted to narcotic pain medications as a result of their post-surgical pain management therapy.

Correlation analyses showed statistically significant associations between patients' anxiety levels about pain before surgery and actual levels of post-surgical painpatients with higher anxiety levels experienced higher levels of pain after surgery than patients who reported lower levels of anxiety about pain before surgery (Figure 1). Pain intensity levels before surgery were 


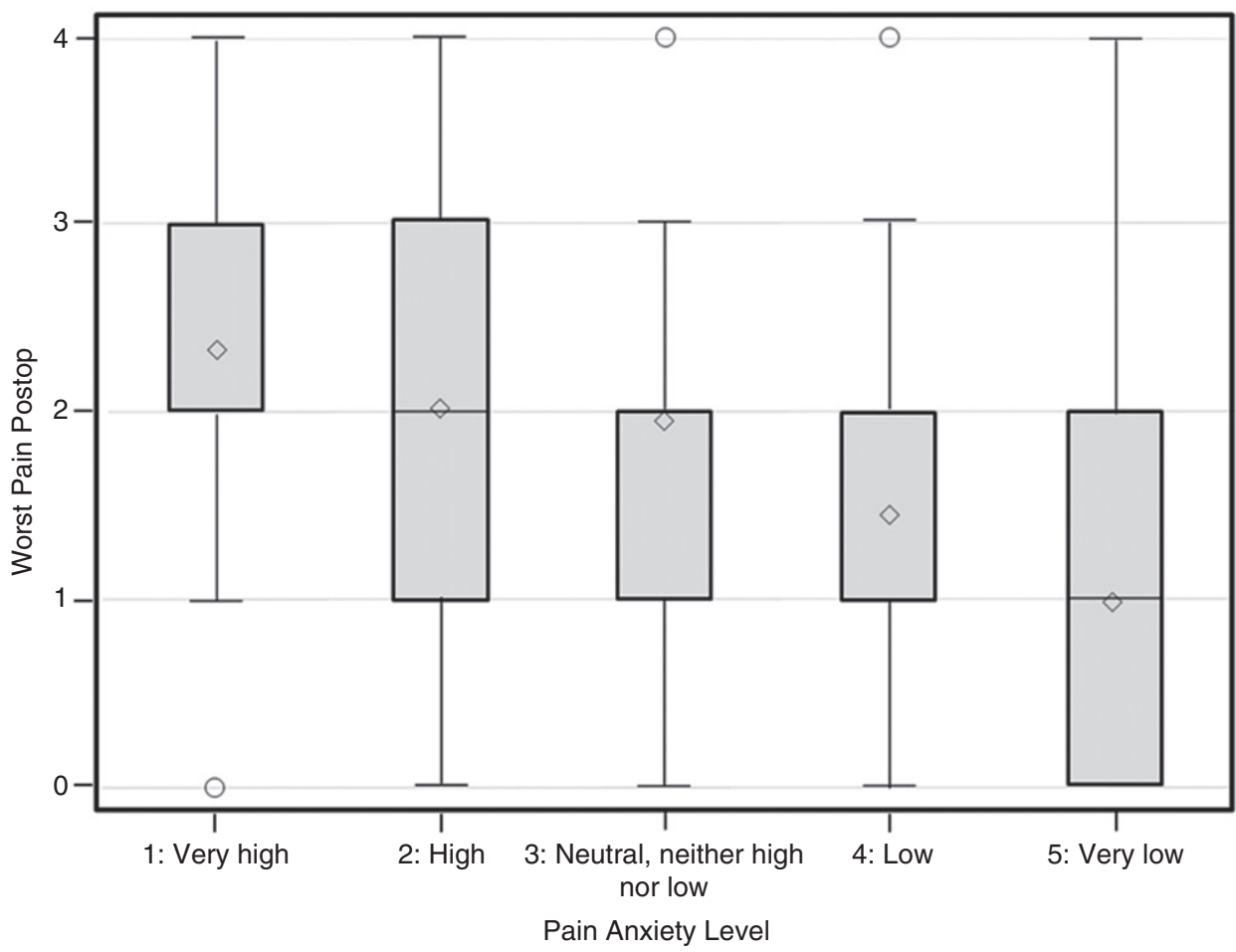

Figure 1. Association between anxiety about pain and post-surgical pain intensity. Kruskal-Wallis chi-square statistic: $34.01 ; p<0.0001$. Shaded bars extend from the 25th percentile (bottom of bar) to the 75th percentile (top of bar). Means are shown by the diamond symbol. Medians equal 2 for the 3 highest anxiety categories, and equal 1 for the 2 lower categories. Vertical lines above and below each bar extend to the limits of the data, except where extreme outliers are shown by circles.

predictive of severity of post-operative pain-greater levels of pre-operative pain were associated with worse post-operative pain (Kruskal-Wallis chi-square statistic: $3.8 ; p=0.003)$.

\section{Discussion}

The design and format of our survey were similar to those conducted $\sim 10$ years ago by Apfelbaum et al. ${ }^{3}$ and 20 years ago by Warfield and Kahn ${ }^{4}$. Based on the results from all three surveys, it seems there has been little or no improvement in post-surgical pain management during the past 2 decades (Figure 2), despite numerous advancements in pain treatment standards. A large majority (86\%) of surgery patients experience post-surgical pain-a rate that has actually increased from 2 decades ago-and three quarters of those patients who did experience post-surgical pain reported its intensity as moderate-to-extreme. Slightly higher proportions of respondents reported moderate-toextreme pain in the earlier surveys; Apfelbaum et al. survey: $86 \%$; Warfield and Kahn survey: $80 \%$. The proportion of patients experiencing pain after hospital discharge was higher in our survey (84\%) than the Apfelbaum et al. survey (75\%); Warfield and Kahn did not assess this outcome. Pain after surgery appears to be an even more prominent concern today ( $80 \%$ in the current survey) than it was 10 years ago (59\%) and 20 years ago (57\%), perhaps reflecting greater awareness of post-surgical pain as reported in the lay press and efforts to highlight pain as the 'fifth vital sign'. Despite high prevalence of intense pain, $90 \%$ of patients $(269 / 300)$ reported satisfaction with their post-surgical pain management. Reasons for this apparent paradox are unclear. Because most patients expect to experience pain after surgery, it is possible that satisfaction levels are more dependent on factors such as attentiveness of the hospital staff, cleanliness of the facility, and food quality, than the physical discomfort associated with surgical intervention. Interestingly, the satisfaction rate in our study was nearly identical to the rate reported a decade ago by Apfelbaum et al. ${ }^{3}$ (88\% satisfied). In light of recent efforts by numerous clinical groups and payors encouraging heightened awareness among clinicians regarding patient education and best practices for pain management, it is not surprising that more patients in the current survey received perioperative pain counseling $(\sim 74 \%)$ than in the previous two surveys $\left(\sim 65 \%\right.$ and $\sim 53 \%$, respectively $\left.{ }^{3,4}\right)$.

Use of non-pharmacologic modalities for pain management also has increased over the past 2 decades from $46 \%$ in the Warfield and Kahn survey to $60 \%$ in the current survey. Morphine, meperidine, and codeine/acetaminophen combinations were the most frequently administered opioid analgesic medications in the previous surveys ${ }^{3,4}$. 


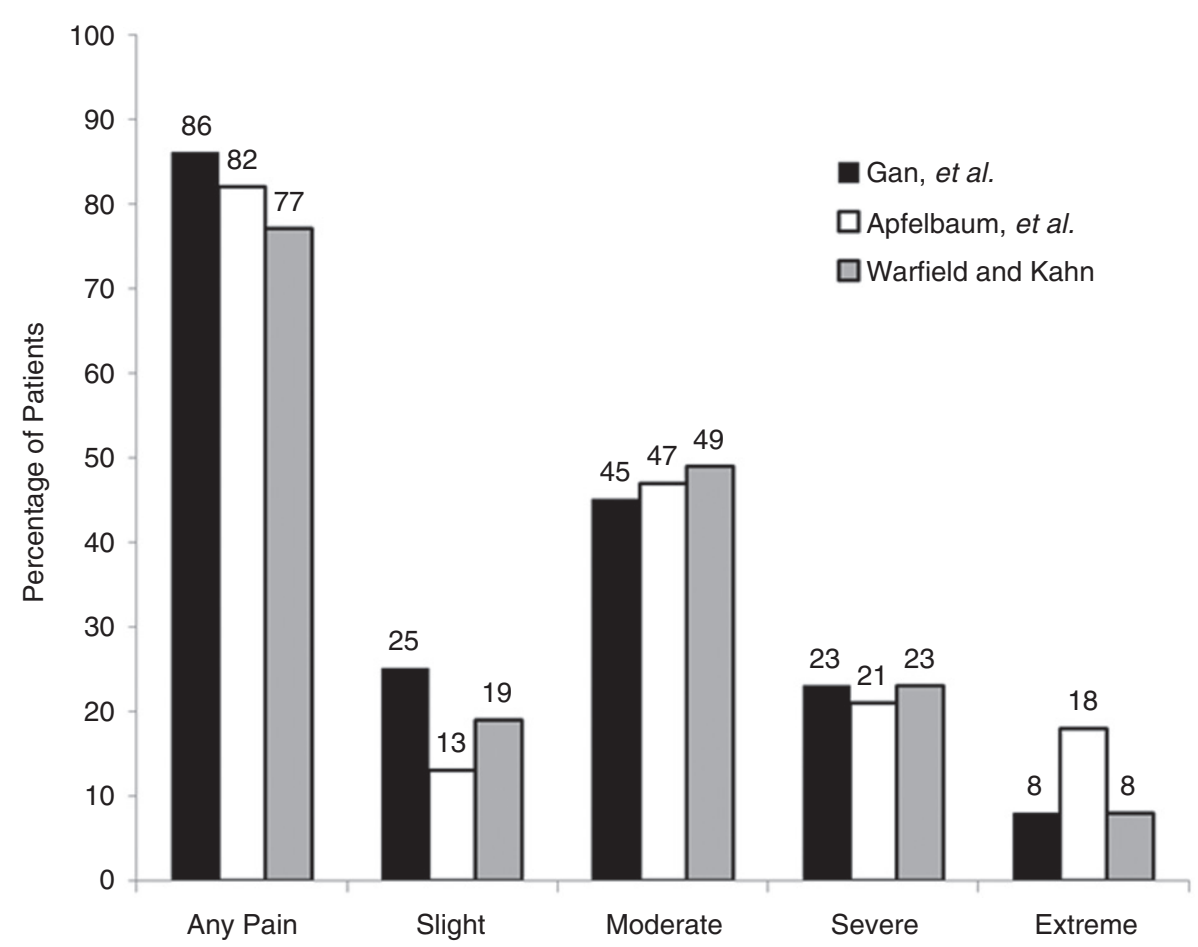

Figure 2. Proportions of patients reporting various post-surgical pain levels in the Warfield and Kahn ${ }^{4}$ survey, the Apfelbaum et al. ${ }^{3}$ survey, and the current survey.

Since then, hydrocodone/acetaminophen and oxycodone/ acetaminophen combinations have emerged as the most commonly used opioid-based analgesics; in the current survey, the proportion of patients receiving these medications was 3-4-times greater than the proportion that received any other opioid. Patient perceptions regarding inevitability of post-surgical pain have changed little over the past 2 decades; $77 \%$ of respondents in the earliest study expressed belief that some pain is necessary after surgery compared with $84 \%$ in the current study.

Our study included several questions not included in the other surveys. We assessed patients' anxiety levels about pain and worries about addiction, and investigated potential associations between pain anxiety and postsurgical pain levels. About half the patients in our survey (46\%) expressed high anxiety before surgery about pain they anticipated after surgery; and we found a significant association between pain anxiety levels and post-surgical pain levels. Although 'anxious' respondents were more likely to experience high pain levels after surgery than respondents without anxiety, association does not imply causation. We found that only about one third of respondents expressed concerns regarding addiction in our survey population.

Overall, incidence and type of adverse effects and level of patient satisfaction with pain management were similar between inpatients and outpatients in our study. High proportions of both inpatients and outpatients experienced pain before and immediately after surgery, as well as after discharge, although the difference between groups was roughly 10 percentage points higher in the inpatient subgroup at all three time points. The incidence and severity of post-surgical pain reported by ambulatory and inpatient respondents are somewhat higher than that reported in other studies. McGrath et al. ${ }^{11}$ conducted a survey of 5703 patients who had undergone ambulatory surgery within $72 \mathrm{~h}$ before their survey, and $55 \%$ reported they had some degree of pain during the first $24 \mathrm{~h}$ after surgery; of these, $48 \%$ had moderate-to-severe pain. In a study of 648 patients undergoing various types of surgery in an ambulatory setting, Gramke et al. ${ }^{12}$ monitored pain intensity levels on a daily basis for 4 days after surgery. In this study, only $26 \%$ of respondents reported moderateto-severe post-surgical pain on the day of surgery, and the proportion decreased to $9 \%$ by post-operative day (POD) 4. In a study of 1490 surgical inpatients, Sommer et al. ${ }^{13}$ also monitored pain levels daily through POD 4. They found that $40 \%$ of their patients experienced moderate-to-severe pain on the day of surgery, with $14 \%$ of patients continuing to report moderate-to-severe pain on POD 4. Reasons for the disparity in pain rates reported in these three studies compared with our study (as well as the Apfelbaum et al. $^{3}$ and Warfield and $\mathrm{Kahn}^{4}$ studies) are unclear. McGrath et al. ${ }^{11}$, Gramke et al. ${ }^{12}$, and Sommer et al. ${ }^{13}$ conducted their pain studies at the time of surgery rather than at later time points. Also, all three of those studies were conducted in non-US populations, which may account for some of the differences across studies. 
The healthcare landscape continues to change-new incentives and benchmarks for institutional performance are being established that tie patient experiences (including pain experiences) within healthcare systems to financial reimbursement rates. The Hospital Consumer Assessment of Healthcare Providers and Systems (HCAHPS) survey is a standardized survey instrument developed by the Centers for Medicare \& Medicaid Services (CMS) and the AHRQ to measure patients' perceptions of their hospital experience ${ }^{14}$. It focuses on 10 key patient-centric topics, including communication about medicines and pain management. This survey has been endorsed by the National Quality Forum (NQF), a national organization that represents stakeholders with shared interest in improving hospital quality. A portion of government-funded reimbursement to healthcare facilities depends on timely and accurate reporting of HCAHPS data; these data are also used by federal healthcare agencies to calculate value-based incentive payments to healthcare institutions. The recently formed PatientCentered Outcomes Research Institute (PCORI) ${ }^{15}$ has been authorized by Congress to investigate benefits and harms of various preventive, diagnostic, therapeutic, palliative, and health delivery system interventions. A key function of PCORI will be to highlight comparisons and outcomes that matter to patients, so that patients can make informed decisions about their healthcare. Based on the results of our study, it appears that healthcare professionals will be challenged to meet the standards that have been set for pain management.

Further, the pain treatment landscape is evolving toward heavier reliance on multimodal analgesic regimens for management of post-surgical pain rather than opioidonly regimens. The goal of a multimodal approach is to provide effective analgesia while reducing the use of opioids and the incidence of opioid-related adverse effects ${ }^{6}$. Studies have shown that use of multimodal analgesic regimens can indeed achieve both effective pain control and a reduction in opioid-related adverse events ${ }^{6,16-18}$. The current American Society of Anesthesiologists practice guidelines for acute pain management in the perioperative setting recommend the use of multimodal analgesic techniques whenever possible ${ }^{6}$.

There are several limitations that should be considered when interpreting the results of this survey. Our survey was not conducted immediately after surgery; thus, participant responses may have been influenced by recall bias. It could also be argued that the average 14-month time span from surgery date to survey date may have resulted in more clinically meaningful responses from survey participants. The time delay may have allowed participants to put their surgery in a proper context. The survey was not designed to capture detailed information regarding types of surgeries conducted and types of intraoperative anesthetics and/or analgesics used. Obviously, these factors could have impacted respondents' post-surgical pain experiences. The study population was relatively small $(n=300)$, slightly larger than the population studied by Apfelbaum et al. ${ }^{3}$ $(n=250)$ and smaller than the one surveyed by Warfield and $\operatorname{Kahn}^{4}(n=500)$. We were unable to account for the potential influence of demographic characteristics on survey results; a large proportion of our study population was white (230/300; 77\%) and female (195/300; 65\%). Also, our results may have been biased by physician respondents' choices regarding which patients they chose for participation in the survey. Despite these limitations, the data obtained from the survey allowed us to gain useful insight on the current state of post-surgical pain management from the patients' perspective.

\section{Conclusion}

In summary, despite the heightened awareness regarding pain and the clinical advancements in pain management during the past 20 years, there has been little improvement in post-surgical analgesia. Most patients continue to experience significant pain following surgery. With the increasing prominence of performance-based reimbursement and publicly-available data regarding patients' experiences during contact with healthcare systems, healthcare professionals will be challenged to find better ways to achieve adequate post-surgical analgesia for their patients.

\section{Transparency}

\section{Declaration of funding}

This survey was supported in part by a research grant from Pacira Pharmaceuticals, Inc. Personnel from Pacira had no role in development of the survey, conducting the study, or analysis of results.

\section{Declaration of financial/other relationships}

Dr Tong Gan has received research funding from AcelRx, Cumberland, Fresenius, Pacira Pharmaceuticals, Inc., and Covidien. Dr Timothy Miller is a consultant for Covidien, Edwards Lifesciences Corporation, and Hospira, Inc., and has received research funding from Cheetah Medical. Dr Ashraf Habib, Mr William White, and Dr Jeffrey Apfelbaum have no relevant conflicts of interest to disclose. CMRO Peer Reviewers on this manuscript have no relevant financial relationships to disclose.

\section{Acknowledgments}

Writing and editorial assistance was provided by Michael Morren, RPh, MBA, of Peloton Advantage, LLC, supported by Pacira Pharmaceuticals, Inc. The authors were fully responsible for the content, editorial decisions, and opinions expressed in the current article. The authors did not receive an honorarium related to the development of this manuscript. 


\section{References}

1. Cullen KA, Hall MJ, Golosinskiy A. Ambulatory surgery in the United States, 2006. Natl Health Stat Report 2009;11:1-25

2. National Center for Health Statistics. Number of all-listed procedures from discharges from short-stay hospitals by procedure category and age: United States, 2009. Atlanta, GA: Centers for Disease Control and Prevention, Available at: http://www.cdc.gov/nchs/data/nhds/4procedures/2009pro4_ numberprocedureage.pdf. Accessed September 12, 2012

3. Apfelbaum JL, Chen C, Mehta SS, et al. Postoperative pain experience: results from a national survey suggest postoperative pain continues to be undermanaged. Anesth Analg 2003;97:534-40

4. Warfield CA, Kahn CH. Acute pain management. Programs in U.S. hospitals and experiences and attitudes among U.S. adults. Anesthesiology 1995;83:1090-4

5. Pain Management Guideline Panel. Clinicians' quick reference guide to postoperative pain management in adults. Rockville, MD: Agency for Health Care Policy and Research, US Department of Health and Human Services. J Pain Symptom Manage 1992;7:214-28

6. American Society of Anesthesiologists Task Force on Acute Pain Management. Practice guidelines for acute pain management in the perioperative setting: an updated report by the American Society of Anesthesiologists Task Force on Acute Pain Management. Anesthesiology 2012;116:248-73

7. The Management of Postoperative Pain Working Group. VHA/DoD clinical practice guideline for the management of postoperative pain. Washington, DC: Veterans Health Administration, US Department of Veterans Affairs/ Department of Defense. May 2002. Available at: http://www.healthquality.va.gov/pop/pop_fulltext.pdf. Accessed August 22, 2012

8. The Joint Commission. Facts about pain management. Oakbrook Terrace, IL: The Joint Commission. January 2012. Available at: http://www.jointcommission.org/pain_management/. Accessed August 21, 2012

9. American Society of Anesthesiologists Task Force on Acute Pain Management. Practice guidelines for acute pain management in the perioperative setting. A report by the American Society of Anesthesiologists Task Force on Pain Management, Acute Pain Section. Anesthesiology 1995;82:1071-81

\section{Appendix: US adult questionnaire}

Q.1. In the past 5 years, have you, personally, had surgery of any type?

Base: Total consumers

Yes (Proceed)

No (Do not proceed)

Q.2a. For your most recent surgical procedure, in what type of facility was the surgery performed?

Base: Consumers who have had surgery in the past 5 years

A hospital as an inpatient

A hospital as an outpatient

A doctor's office

An outpatient clinic

A free-standing surgicenter

Q.2b. What type of procedure did you have?
10. American Society of Anesthesiologists Task Force on Acute Pain Management. Practice guidelines for acute pain management in the perioperative setting: an updated report by the American Society of Anesthesiologists Task Force on Acute Pain Management. Anesthesiology 2004;100:1573-81

11. McGrath $B$, Elgendy $H$, Chung $F$, et al. Thirty percent of patients have moderate to severe pain $24 \mathrm{hr}$ after ambulatory surgery: a survey of 5703 patients. Can J Anaesth 2004;51:886-91

12. Gramke HF, de Rijke JM, Van Kleef M, et al. The prevalence of postoperative pain in a cross-sectional group of patients after day-case surgery in a university hospital. Clin J Pain 2007;23:543-8

13. Sommer M, de Rijke JM, Van Kleef M, et al. The prevalence of postoperative pain in a sample of 1490 surgical inpatients. Eur J Anaesthesiol 2008;25:267-74

14. Centers for Medicare \& Medicaid Services and the Agency for Healthcare Research. Hospital Consumer Assessment of Healthcare Providers and Systems survey fact sheet (CAHPS hospital survey). Rockville, MD: US Department of Health and Human Services. May 2012. Available at: http:// www.hcahpsonline.org/files/

HCAHPS\%20Fact\%20Sheet\%20May\%202012.pdf. Accessed September 26, 2012

15. Patient-Centered Outcomes Research Institute. Patient-centered outcomes research. Washington, DC: Patient-Centered Outcomes Research Institute (PCORI), April 2012. Available at: http://www.pcori.org/what-we-do/pcor/ Accessed May 13, 2013

16. Buvanendran A, Kroin JS. Multimodal analgesia for controlling acute postoperative pain. Curr Opin Anaesthesiol 2009;22:588-93

17. Marret E, Kurdi O, Zufferey P, Bonnet F. Effects of nonsteroidal antiinflammatory drugs on patient-controlled analgesia morphine side effects: meta-analysis of randomized controlled trials. Anesthesiology 2005:102:1249-60

18. Buvanendran A, Kroin JS, Tuman KJ, et al. Effects of perioperative administration of a selective cyclooxygenase 2 inhibitor on pain management and recovery of function after knee replacement: a randomized controlled trial. JAMA 2003;290:2411-8

Q.3. Before undergoing the surgery, were you concerned about: (multiple selections allowed)

Base: Consumers who have had surgery in the past 5 years Whether the surgery would actually improve your condition?

How you would be treated by the doctors, nurses, or other healthcare professionals?

The pain you might experience during surgery?

The pain you might experience after surgery?

Whether you would fully recover from the surgery?

Q.4. What level of anxiousness or nervousness did you have about the pain you would experience after surgery?

Very high

High

Neutral, neither high nor low 
Low

Very low

Q.5. Following your surgery, that is, immediately afterwards and up to 2 weeks following surgery, did you experience any pain?

Base: Consumers who have had surgery in the past 5 years

$$
\text { Yes }
$$

No

Q.6. Which of the following describes the highest degree of pain you felt up to 2 weeks after surgery?

Base: Consumers who have had surgery in the past 5 years, and who experienced pain immediately afterwards and up to 2 weeks following their surgery
Slight pain
Moderate pain
Severe Pain
Extreme Pain
$\mathrm{DK} / \mathrm{NA}$

Q.7. Did you receive any medication for your pain within 2 weeks after surgery?

Base: Consumers who have had surgery in the past 5 years
Yes
No
DK/NA

Q.8. What medication or medications did you receive? Base: Consumers who have had surgery in the past 5 years, and who received medication for their pain within 2 weeks after surgery (multiple selections allowed)
Morphine
Demerol
Dilaudid
Vicodin
Norco
Percocet
Oxycodone
Hydrocodone
Darvon
Darvocet
Aspirin
Motrin
Toradol
Tylenol
Tylenol (with codeine)
Indocin
Local anesthetic
Other
DK/NA

Q.9. How did you receive pain medication?

Base: Consumers who have had surgery in the past 5 years, who are unsure of the medication(s) they received during the 2-week period after surgery (multiple selections allowed)

$\square$ Injection

$\square$ Intravenous

$\square$ Patient-controlled IV pump

$\square$ PainBuster pump

$\square$ Spinal/epidural

Oral/tablet form

Q.10. Do you know whether the drug or drugs were narcotic or non-narcotic?

Base: Consumers who have had surgery in the past 5 years, who are unsure of the medication(s) they received during the 2 -week period after surgery

$\square$ Narcotic

$\square$ Non-narcotic

$\square$ Both

DK/NA

Q.11. Did you (with regard to medication):

Base: Consumers who have had surgery in the past 5 years, and who received medication for pain within 2 weeks after surgery

$\square$ Have to ask for pain medication?

$\square$ Receive pain medication on schedule?

$\square$ Have to wait for pain medication when you asked for it?

$\square$ Control your own pain medication with an IV pump?

Q.12. After the first dose of pain medication, how much pain did you have? Would that be:

Base: Consumers who have had surgery in the past 5 years, and who received medication for pain within 2 weeks after surgery
$\square$ None
$\square$ Slight
$\square$ Moderate
$\square$ Severe
$\square \mathrm{DK} / \mathrm{NA}$

Q.13. Did you experience any side-effects from any of your pain medications?

Base: Consumers who have had surgery in the past 5 years, and who received medication for pain within 2 weeks after surgery

$\square$ Yes

$\square$ No

Q.14. What side-effects did you experience?

Base: Consumers who have had surgery in the past 5 years, and who have experienced some type(s) of side effect(s) from their pain medication (multiple selections allowed)

$\square$ Confusion

Drowsiness 
Nausea

Vomiting

Itching

Difficulty urinating

Sleeplessness

Mood changes

Dizziness

Constipation

Slow or troubled breathing

Other

DK/NA

Q.15. Did you use any of the following non-drug treatments for your pain after surgery?

Base: Consumers who have had surgery in the past 5 years (multiple selections allowed)

$\square$ Relaxation techniques

Biofeedback techniques

Application of heat

Application of cold

Massage

Exercise

Acupuncture

Transcutaneous electrical nerve stimulation (TENS)

None

DK/NA

Q.16. Prior to surgery, did someone from the facility where you had surgery talk with you about how your pain would be treated?

Base: Consumers who have had surgery in the past 5 years Yes

No

Q.17. Who talked with you?

Base: Consumers who have had surgery in the past 5 years who, prior to surgery, talked with someone about how their pain would be treated (multiple selections allowed)

Anesthesiologist

Nurse

Social worker

Surgeon

Other type of doctor

DK/NA

Q.18. Did you have pain before surgery?

$\square$ Yes

$\square$ No

DK/NA

Q.19. What was your worst pain before surgery?

None

Slight

Moderate

Severe

DK/NA
Q.20. Within 5 days after your operation, did someone ask you if you had pain?

Base: Consumers who have had surgery in the past 5 years

$\square$ Yes

$\square$ No

$\square$ DK/NA

Q.21. Who asked you?

Base: Consumers who have had surgery in the past 5 years, and who were asked if they had any pain within 5 days after their operation (multiple selections allowed)

$\square$ Anesthesiologist

$\square$ Nurse

$\square$ Patient representative

$\square$ Social worker

$\square$ Surgeon

$\square$ Other type of doctor

Q.22. How satisfied were you with your overall pain management after your surgery?

$\square$ Very satisfied

Somewhat satisfied

$\square$ Neither satisfied nor dissatisfied

$\square$ Somewhat dissatisfied

Very dissatisfied

Q.23. Have you ever refused or postponed surgery because you were worried about the possibility of experiencing pain?

Base: Total consumers

$\square$ Yes

$\square$ No

$\square$ DK/NA

Q.24. Do you believe it is necessary to experience some pain after surgery?

Base: Total consumers

$\square$ Yes

$\square$ No

DK/NA

Q.25. Do you believe it's okay for patients to complain about pain after surgery?

Base: Total consumers

$\square$ Yes

$\square$ No

$\square \mathrm{DK} / \mathrm{NA}$

Q.26. Which of the following do you believe would provide stronger relief from pain after surgery? Pain relief medication given:

$\square$ By injection

$\square$ By IV

$\square$ In tablet form

$\square$ Pain pump

$\square$ By spinal injection (epidural)

They are about the same 
Depends on the surgery (volunteered)

DK/NA

Q.27. Do you think there are some pain medications prescribed after surgery that cause side effects?

Base: Total consumers

$\square$ Yes

$\square$ No

$\square \mathrm{DK} / \mathrm{NA}$

Q.28. What side-effects are caused by pain medications prescribed after surgery?

Base: Consumers who believe that some pain medications prescribed after surgery cause side-effects

Q29a. Did you take any over-the-counter pain medication after surgery?

$\square$ Yes

No

DK/NA

Q.29b. What over-the-counter pain medication did you take?

Q.30. If your doctor gave you the choice of a narcotic or a non-narcotic pain reliever after surgery, which would you choose?

Base: Total consumers

Narcotic

Non-narcotic

Neither/none (volunteered)

Either/doesn't matter (don't care [volunteered])

DK/NA

Q.31. Are you worried about becoming addicted to the pain medications after surgery?
Yes
No
DK/NA

Q.32. How would you rate your pain threshold?

Very high

High

Neutral, neither high nor low

Low

$\square$ Very low

Q.33. How would you rate your anxiety level?

Very high

High

Neutral, neither high nor low

Low

Very low

Q.34. What is your marital status?

Base: Total consumers
Single, never married

Married

Separated

Widowed

Divorced

Refused

Q.35. Would you consider yourself to be the male head of this household?

Base: Male consumers

$\square$ Yes

$\square$ No

Q.36. Would you consider yourself to be the female head of this household?

Base: Female consumers

$\square$ Yes

$\square$ No

Q.37. What is your age?

$\square$ 18-29

$\square 30-39$

$\square$ 40-44

$\square 45-54$

55-64

$\square 65-74$

75 or over

Refused

Q.38. What is the last grade of school you completed?

Base: Total consumers

Less than high school graduate

$\square$ High school graduate

$\square$ Technical school/other

Some college

$\square$ Graduated college

$\square$ Graduate school or more

Refused

Q.39. What is your total annual household income from all sources and before taxes?

Base: Total consumers

$\square$ Less than $\$ 10,000$

$\square 10,000$ but less than $\$ 15,000$

$\square \$ 15,000$ but less than $\$ 20,000$

$\square \$ 20,000$ but less than $\$ 25,000$

$\square \$ 25,000$ but less than $\$ 30,000$

$\square \$ 30,000$ but less than $\$ 40,000$

$\square \$ 40,000$ but less than $\$ 50,000$

$\$ 50,000$ but less than $\$ 75,000$

$\square \$ 75,000$ but less than $\$ 100,000$

$\square 100,000$ or over

Refused

Q.40. Would you consider yourself to be white, Hispanic, African American, Asian, or of some other race?

Base: Total consumers

White

Hispanic 
African American

Asian

$\square$ Mixed race

$\square$ Other

$\square$ Refused

Q.41. Gender

Base: Total consumers

$\square$ Male

$\square$ Female
Q.42. How much would you pay out-of-pocket to be completely pain-free after surgery?

$\$$

Q.43. How much would you pay if an effective pain medication would not give you any side-effects, such as nausea, vomiting, itchiness, constipation, or drowsiness?

$\$$ 